\title{
Numerical study on a relationship between scattering seismic wave and crustal stress field, and its possibility of application to field data
}

\author{
Kyosuke OKAMOTO ${ }^{1}$, Hitoshi MIKADA ${ }^{2}$, Tada-nori GOTO², and Junichi TAKEKAWA ${ }^{2}$ \\ ${ }^{1}$ Dept. of Civil and Earth Res. Eng., Kyoto University and JSPS Research Fellow \\ ${ }^{2}$ Dept. of Civil and Earth Res. Eng., Kyoto University
}

\begin{abstract}
Coda- $Q$ is a stochastic parameter reflecting the heterogeneities of medium that seismic waves travel through. We investigate a relationship between crustal stress field and the coda- $Q$. In this study we employ a 2-D Finite Different Method to calculate seismic wave propagation through the lower and upper crust. We confirmed that the coda- $Q$ would vary with the stress loaded to an elastic medium using the numerical simulation. The coda- $Q$ roughly shows a proportional relationship with magnitude of the stress. We also confirmed that the upper crust, where consists of a crack-rich medium, affects the coda- $Q$ dominantly. Next, a relationship between coda- $Q$ obtained from actual seismic records and crustal strain obtained from GPS data is examined, and they mark high correlation coefficient. The filed example also supports that coda- $Q$ has a correlation with crustal stress.
\end{abstract}

\section{INTRODUCTION}

In research of earthquake occurrence, it is important to monitor the change of crustal stress field in the seismogenic zones. However, it is difficult to measure the stress at seismogenic depth because of limitation of equipment ability, cost of drilling, and so on.

Recently, Hiramatsu et al. $(2010)^{1)}$ stressed that coda- $Q$ (denoted as $Q_{c}$, hereafter) is a parameter of the differential strain rate, which relates with the stressing rate of shear stress, from the observation of the $Q_{c}$ in the vicinity of the 1995 Southern Hyogo Prefecture earthquake before and after the main shock event. Sugaya et al. (2009) ${ }^{2)}$ discussed a change in $Q_{c}$ after the 1995 Southern Hyogo Prefecture earthquake in connection with a property change in the crust, particularly in the lower crust. The cracks opened or closed by the stress change are one of the major factors which change the property of the crust. Jin et al. (2004) ${ }^{3)}$ and Aki $(2004)^{4)}$ proposed a "Brittle-Ductile Hypothesis" after a long-term observation of the seismicity around the San Andreas Fault that has led them to find a high correlation with $Q_{c}$.

The parameter $Q_{c}$ is derived from attenuation ratio of energy in a coda wave, which origin is residual vibration caused by inhomogeneities around the seismic source. Some papers reported anomalous value of $Q_{c}$ around volcanoes, faults ${ }^{5)}$, which means the media has anomalous inhomogeneity. Also the recent results ${ }^{4)}$ reported that anomalous value of $Q_{c}$ was detected after a large earthquake. These results suggest that the order of inhomogeneities may vary in the course of long-term earthquake generation cycle, i.e., before and after the failure of crustal material takes place. Here we make a reasonable deduction with fair certainly that the stochastic parameter $Q_{c}$ can have a certain relationship with physical or state properties of the stress field in the particular part of the crust where seismic waves propagate through, since the change in the stress filed is a trigger of earthquakes.

In this paper, we employ numerical simulation schemes to test our hypothesis that $Q_{c}$ would vary with change in the stress acting on the medium where seismic waves travel through. For this objective, we have already examined effect of elastic displacement of the cracks, which is induced by the loaded stress, on $Q_{c}{ }^{6}$. Also effect of anisotropy on elastic wave velocity field and change in crack orientation is examined in Okamoto et al. $(2011)^{7}$. Then it has been revealed that anisotropy on elastic wave velocity field and change in orientation of crack are dominant effects affecting $Q_{c}$. We reveal a relationship between $Q_{c}$ and crustal stress, considering these two dominant effects using a numerical simulation. Also we consider if there is a relationship between $Q_{c}$ calculated from field data and the strain obtained by GPS measurement, which is considered as a proxy of the crustal stress at near surface. 


\section{NUMERICAL SIMULATION}

We employ a 2-D Finite Different Method (FDM) with discontinuous grid to simulate propagating and scattering elastic waves in the crust. Equation of motion (Eq. 1) and equation of stress-strain relation (Eq. 2 and 3) for 2-D SH wave are discretized by a rotated staggered grid $^{8)}$.

$$
\begin{gathered}
\rho \frac{\partial^{2} u_{y}}{\partial t^{2}}=\frac{\partial \tau_{x y}}{\partial x}+\frac{\partial \tau_{y z}}{\partial z} \\
\tau_{x y}=\mu(\theta) \frac{\partial u_{y}}{\partial x} \\
\tau_{y z}=\mu(\theta) \frac{\partial u_{y}}{\partial z}
\end{gathered}
$$

where $\rho$ is density, $u$ is displacement, $\tau$ is stress, $\mu(\theta)$ is the Lame's constant varying with propagating angle $\theta, t$ is time and $x, y$ and $z$ are special coordinate.

Fig. 1 shows a simulation model. The upper crust is discretized by the FDM mesh which interval is three times as fine as that of the lower crust. Using this mesh, detailed shape of the cracks in the upper crust, where larger cracks distribute than in the lower crust, can be described. We assume that plane wave incidents from the bottom of the lower crust. A wave form of the plane wave is the Ricker wavelet with the central frequency of $9 \mathrm{~Hz}$. We assume that the initial model is stable under the initial confining pressure at the corresponding depth. In addition with the initial confining pressure, normal stress whose magnitude ranges from $-4 \mathrm{MPa}$ to $16 \mathrm{MPa}$ is loaded along the $x$ or $z$ axis. The cracks close or open selectively by the compressive or tensile stress respectively, and then deflector arrangement of the cracks and anisotropy on elastic wave velocity filed occur ${ }^{9)}$ in the upper crust. The cracks satisfying Eq. 4 close when the compression is loaded and the cracks satisfying Eq. 5 newly

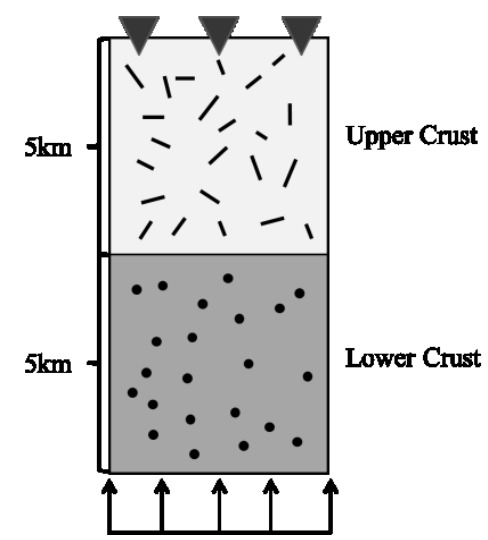

Fig. 1: Incident wave comes from the bottom of the model. 600 cracks having unique shape distribute in the upper crust and 1,000 point shaped scatterers distribute in the lower crust. open when the tension is loaded.

$$
\begin{aligned}
& \alpha \leq \sigma_{n} / E \\
& \alpha>\sigma_{n} / E
\end{aligned}
$$

where $\alpha$ is crack aspect ratio, $\sigma_{n}$ is magnitude of stress normal to a crack and $E$ is the Young's module. The number of newly opening cracks due to the tension is same as the number of closed cracks when the same magnitude of compressive stress is loaded. These equations are obtained by a rock experiment. However, here we assume that they can be applied to larger scale, the crust. We also consider new opening cracks at the edges of the closed cracks due to incensement of deviatoric stress ${ }^{10)}$. Length of the new opening cracks is far shorter than that of the original crack.

A seismogram is recorded at the stations located on the surface of the model before and after the stress is loaded. From this seismogram, $Q_{c}^{-1}$ (the reciprocal of $Q_{c}$ ) is calculated after fitting Eq. 6 to envelope of the coda wave $e(t)$.

$$
e(t)=A_{0} t^{-n} \exp \left(-\frac{\omega}{2 Q_{c}} t\right)
$$

where $A_{0}$ is initial amplitude, $t$ represents the time from origin of an earthquake and $\omega$ is angular frequency. The term of $t^{-n}$ represents geometrical spreading. Power $n$ is from 1 to 2 depending on the dominance wave, i.e., surface, diffusive or body waves, in practice. However in this study we set $n=$ 0 because a plane wave, which does not spread, is assumed as the incident wave. Higher $Q_{c}^{-1}$ is observed when the coda wave strongly attenuates.

\section{VARIATION IN $Q_{c}^{-1}$ AGAINST CRUSTAL STRESS}

Fig. 2 shows variation in $Q_{c}^{-1}$ against magnitude of the loaded stress. The $Q_{c}^{-1}$ increases in both cases, when the stress is loaded along the $x$ or $z$ axis. However magnitude range where the $Q_{c}^{-1}$ increases is different between the case of the $x$ and $z$ axes

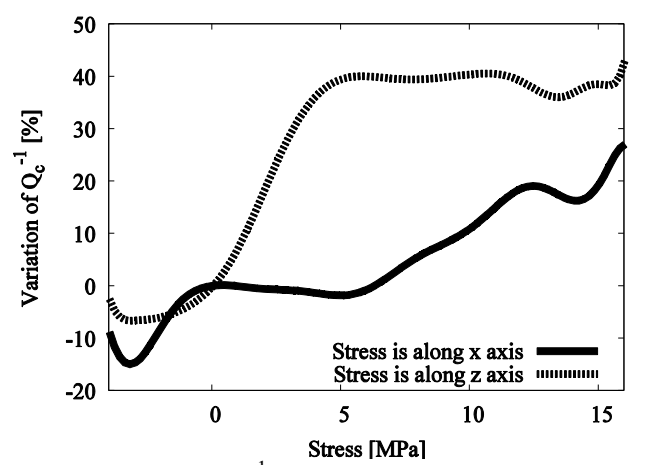

Fig.2: Variation in $Q_{c}^{-1}$ against magnitude of the loaded stress. The stress is loaded along $x$ or $z$ axis. $Q_{c}^{-1}$ at $0 \mathrm{MPa}$ is a standard value for the variation ratio. 


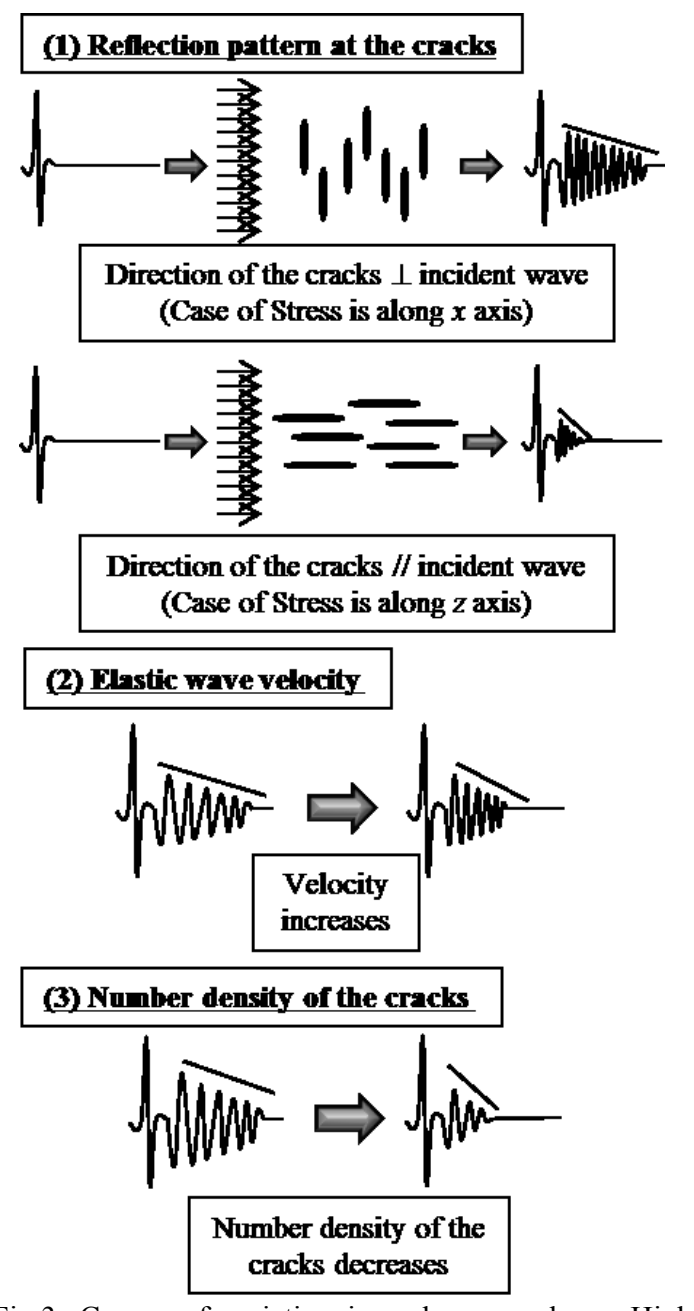

Fig.3: Causes of variation in coda wave shape. High attenuation of the coda wave makes higher value of $Q_{c}^{-1}$.

loading. This increase is due to change in (1) reflection pattern at the cracks, (2) elastic wave velocity field and (3) number density of the cracks (see Fig.3). Little energy in the incident wave transfers to the coda wave when direction of a crack coincides with the incident wave (the case of the stress is loaded along the $z$ axis). It makes the coda wave attenuate and high $Q_{c}^{-1}$ is observed. Also high $Q_{c}^{-1}$ is observed when elastic velocity is faster because the coda wave attenuates much rapidly against time. When number density of the cracks decreases, high $Q_{c}^{-1}$ is also obtained because little energy transfers to the coda wave by scattering from the incident wave. The $Q_{c}^{-1}$ increases due to the effect of (1), (2) and (3) when the stress is loaded along $z$ axis, while due to the effect of (2) and (3) when the stress is loaded along $x$ axis. This discrepancy makes the different tendency of increase in the $Q_{c}^{-1}$ between the $x$ and $z$ axes loading. In brief conclusion, $Q_{c}^{-1}$ increases when the stress is loaded regardless its direction but the magnitude range within that the $Q_{c}^{-1}$ increases has dependence

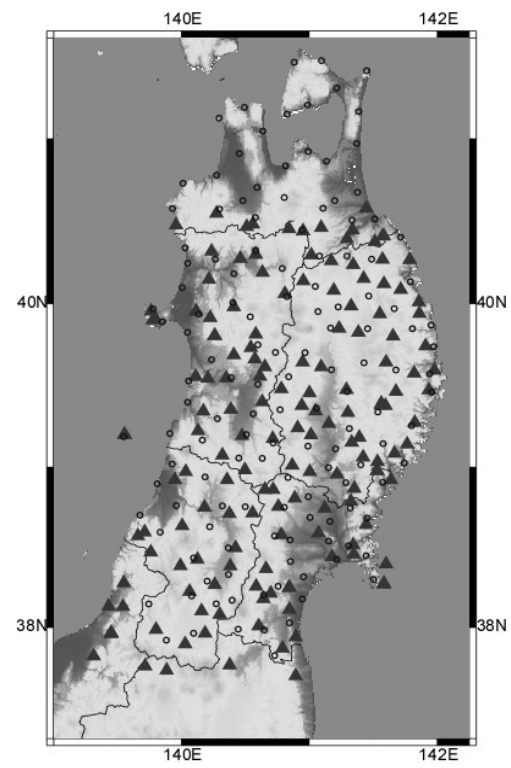

Fig.4: Geometry of Hi-net stations $(\boldsymbol{\Delta})$ and GEONET stations $(\bigcirc)$. is is the analyzed area.

on direction of the loading. $Q_{c}^{-1}$ increases when the confining pressure increases.

\section{FILED DATA ANALYSIS}

It has been revealed that there is a relationship between $Q_{c}^{-1}$ and the crustal stress, using the numerical simulation. We would like to confirm this relationship in filed data. In a field, the crustal stress is difficult to measure as mentioned in the introduction. So that the surface strain measured by GPS is used as a proxy of the crustal stress in this study. The surface strain is calculated using displacements recorded at three GPS stations, which form a triangle array. The analyzed area is chosen around Ishinomaki, Miyagi, shown in Fig.4. The used seismic data are summarized as below, recorded by Hi-net ${ }^{11)}$.

- Observation term: Jul., 2002 - Jan. 2012 (with the SMA, interval is 3 months)

- Distance between an epicenter and the receiver: With in $40 \mathrm{~km}$

- Magnitude range: $M_{w} 1.5-7.0$

- Depth of hypocenter: Shallower than $100 \mathrm{~km}$

A seismogram is filtered by octave-band pass, which range is $4-8 \mathrm{~Hz}$ and smoothed using Route Mean Squared (RMS) technique. Three GEONET stations $^{12)}$ for calculating the surface strain are selected as their triangle includes the all hypocenters used to estimate the $Q_{c}{ }^{-1}$. Large steps in the strain like co-seismic dislocation are removed.

Fig. 5a is time variation in the $Q_{c}^{-1}$ and the surface strain, and Fig. 5b, c, and $\mathrm{d}$ are their correlation at different terms. A reasonable high correlation coefficient is marked at each observation term. So that it is confirmed that the 


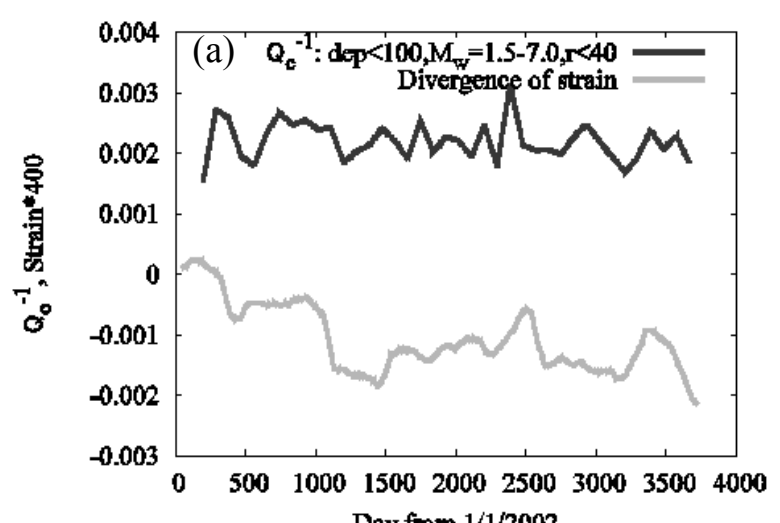

Day from $1 / 1 / 2002$

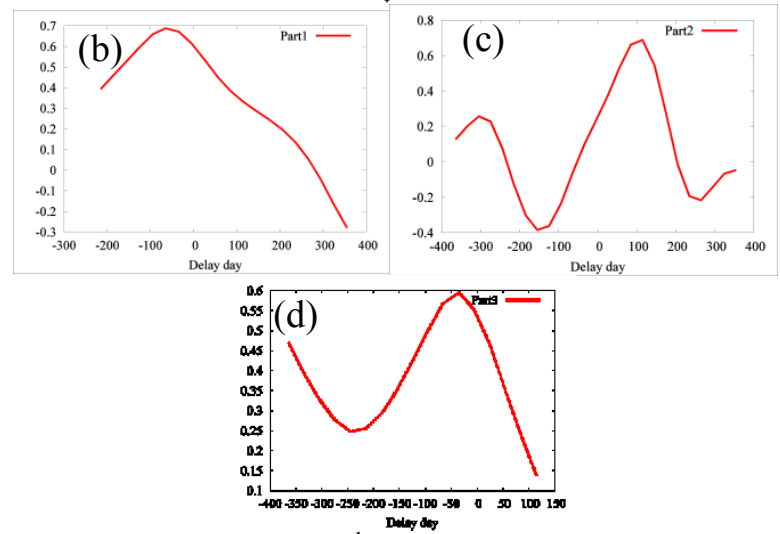

Fig. 5: Variation in the $Q_{c}^{-1}$ and the surface strain. They are correlated, dividing the term into three parts by lapsed days (b) $270-1230$, (c) $1800-2700$, and (d) $2600-3600$.

$Q_{c}^{-1}$, which is seemed to indicate change in the stress field between an epicenter and the receiver, has correlation with the surface strain that relates with change in the stress at the surface of the upper crust. We conclude that a certain relationship can be seen in filed data as revealed by the numerical simulations. However, further studies are needed to reveal a detailed relationship between the $Q_{c}^{-1}$ and the crustal stress in field data.

\section{SUMMARY}

A relationship between $Q_{c}^{-1}$ and the crustal stress filed has been revealed using the numerical simulations. They have a proportional relationship at certain magnitude range. We also revealed a correlation between $Q_{c}^{-1}$ and the surface strain, which can be used as a proxy of the crustal stress. We confirmed that a similar certain relationships between them in filed data.

ACKNOWLEDGMENT: We thank Japan Society for the Promotion of Science (JSPS) for the Grant-in-Aid for JSPS Fellows $(24 \cdot 373)$ for supporting the present research. We also thank National Research Institute for Earth Science and Disaster Prevention (NIED) and Geospatial Information Authority of Japan (GSI) to make their data available.

\section{REFERENCES}

1) Hiramatsu, Y., K., Iwatsuki, S., Ueyama, and T., Iida, 2010, Spatial variation in shear wave splitting of the upper crust in the zone of inland high strain rate, central Japan, Earth Planets Space, 62, 675-684.

2) Sugaya, K., Y., Hiramatsu, M., Furumoto, and H., Katao, 2009, Coseismic change and recovery of scattering environment in the crust after the 1995 Hyogo-ken Nanbu Earthquake, Japan, Bull. Seism. Soc. Am., 99, 435-440.

3) Jin, A., K., Aki, Z., Liu, and V. I.,Keilis-Borok, 2004, Seismological evidence for the brittle-ductile interaction hypothesis on earthquake loading, Earth Planets Space, 56, 823-830.

4) Aki, K., 2004, A new view of earthquake and volcano precursors, Earth Planets Space, 56, 689-713.

5) Matsumoto, S., and Hasegawa, A., 1989, Two-dimensional coda Q structure beneath Tohoku, NE Japan, Geophys. J. Int., 99, 101-108.

6) Okamoto, K., H., Mikada, T., Goto, J., Takekawa, and K., Onishi, 2010, Relation between Coda- $Q$ and stress loaded to an elastic body -parameters of material conditions derived by stochastic measurement: Effect of elastic displacement-, Butsur-tansa (Explor. Geophys), 63, 519-529 (in Japanese with English abstract).

7) Okamoto, K., H., Mikada, T., Goto, and J., Takekawa, 2011, Numerical studies on stress field monitoring using Coda- $Q, S E G$ Expanded Abs., 30, 4229-4233.

8) Saenger, E. H., N., Gold, and S. A., Shapiro, 2000, Modeling the propagation of elastic waves using a modified finite-difference grid, Wave Motion, 31, 77-92.

9) Nur, A., 1971, Effect of Stress on Velocity Anisotropy in Rocks with Cracks, J. Geophys. Res., 76, 2022-2034.

10) Sato, Y., 1992, Theoretical study on dilatancy of dry crack, J. Soc. Mat. Sci., Japan, 41, 1068-1074 (in Japanese with English abstract).

11) Okada, Y., Kasahara, K., Hori, S., Obara, K., Sekiguchi, S., Fujiwara, H., and Yamamoto, A., 2004, Recent progress of seismic observation networks in Japan-Hi-net, F-net, K-NET and KiK-net-, Earth Planets Space, 56, 15-28.

12) Miyazaki, S., Y., Hatanaka, T., Sagiya, and T., Tada, 1998, The Nationwide GPS array as an Earth observation system, Bull. Geogr. Surv. Inst., 44, 11-22. 